Relations industrielles

Industrial Relations

\title{
Daigle, Gérard, dir. et Guy Rocher, coll., Le Québec en jeu : comprendre les grands défis
}

\section{Bernard Solasse}

Volume 49, numéro 3, 1994

URI : https://id.erudit.org/iderudit/050966ar

DOI : https://doi.org/10.7202/050966ar

Aller au sommaire du numéro

Éditeur(s)

Département des relations industrielles de l'Université Laval

ISSN

0034-379X (imprimé)

1703-8138 (numérique)

Découvrir la revue

Citer ce compte rendu

Solasse, B. (1994). Compte rendu de [Daigle, Gérard, dir. et Guy Rocher, coll., Le Québec en jeu : comprendre les grands défis]. Relations industrielles / Industrial Relations, 49(3), 611-613. https://doi.org/10.7202/050966ar

Tous droits réservés @ C Département des relations industrielles de l'Universite Laval, 1994
Ce document est protégé par la loi sur le droit d'auteur. L’utilisation des services d'Érudit (y compris la reproduction) est assujettie à sa politique d'utilisation que vous pouvez consulter en ligne.

https://apropos.erudit.org/fr/usagers/politique-dutilisation/ 
calculations are then carried out to see how long a strike is worthwhile from the union's viewpoint. The chapter by Richardson and Rubin uses a variety of information sources to examine the nature of the management response to the 1989-90 engineering industry strike concerned with reducing the length of the working week. Their work suggests that management was relatively successful in offsetting the costs of a shorter week by bringing about changes in working practices. The final chapter, which is listed as an appendix, contains a wealth of time series strike information for the UK; specifically new information is presented on a per union member, as opposed to per employee, basis.

With strike levels being well down in advanced industrialised economies, the topic of industrial disputes is not a particularly fashionable or hot one at the moment. This being said, there are many positive things to say about this particular book. Important, enduring, questions are examined, original research is presented, the level of analysis is relatively high, and differing discipline and methodological perspectives and approaches are present. Indeed an 'outsider' who still thinks of UK industrial relations research as being all about single plant, qualitative case studies, would particularly benefit from looking at this book; it would indicate just how much things have changed.

One of my few quibbles with the book would be its title. Useful perspectives, varjed perspectives, worthwhile perspectives? Certainly! New perspectives? Well, maybe it's just a question of semantics.

P. B. Beaumont

\section{University of Glasgow}

Le Québec en jeu : comprendre les grands défis, sous la direction de Gérard DAIGLE avec la collaboration de Guy RoCHER, Montréal, Presses de l'Université de Montréal, 1992, 811 p., ISBN 2-76-06-1594-4.

Pas moins de huit cent onze pages publiées serrées; vingt-huit chapitres regroupés en quatre parties répondant aux titres suivants: «L'Économie et le travail», « Le Social », «Le culturel » et «Le Polique ». Quatre parties soit, mais pourquoi pas une cinquième partie qui aurait traité des «enjeux » et des «défis » d'ordre économique, diplomatique, militaire que posent au Québec d'aujourd'hui son insertion et son appartenance « de fait " à la communauté internationale si dissonante et conflictuelle soitelle ? Faut-il retenir ici le manque de ressources ou faire état d'une approche quelque peu « égocentrique » en ce qu'elle resterait centrée sur la recherche d'une identité collective toujours circonscrite?

Vingt-huit chapitres - ou plutôt vingt-huit articles, nous y reviendrons - dont Gérard Daigle, le maître d'œuvre en les circonstances, nous dit dans l'avant-propos qu'il a rédigé et signé, qu'il s'agit de « textes originaux et intégraux » et non pas « d'extraits d'articles ou de volumes déjà publiés » (p. 19). Il reste, sans démentir ni Gérard Daigle, ni les auteurs de ces vingt-huit chapitres couvrant l'ensemble de la période allant des années soixante à quatre-vingt-douze, tel était l'objectif assigné (p. 17), laissent le lecteur attentif sur une impression de «déjà lu » ou de «déjà entendu ». 
Enchaînons : vingt-huit chapitres relevant de «champs d'intérêt » et de « disciplines » diversifiés et faisant de surcroit référence à des problématiques et à des méthodologies elles aussi fort diverses. Comment dès lors en rendre compte dans leur ensemble et d'une façon simplement honnête et respectueuse de leur spécificité? Notre temps n'est plus celui des « Encyclopédistes » ni même celui de «L'honnête homme » qui, en référence à une formation générale et de bon aloi, pouvait prétendre prendre la mesure et se prononcer sur tout. Tel est l'un des effets qu'entraîne la "spécialisation ». Il reste, cependant, que les auteurs de la plupart de ces vingt-huit chapitres se sont efforçés d'annexer à chacune de leur contribution une bibliographie précise qu'apprécieront ceux qui sont appelés à œuvrer au titre d'enseignants ou de chercheurs dans les domaines concernés.

«Avec la collaboration de Guy Rocher ». Cette collaboration se résume apparamment et vraisemblablement au seul « chapitre 15 » qui s'intitule, nous citons : « Autour de la langue : crise et débats, espoirs et tremblements ", soit vingt-huit pages d'une prudence extrême et qui ne dément pas celle dont Guy Rocher faisait preuve, en 1973, lorsqu'il a publié chez HMH Le Québec en mutation que nous avons relu. Une extrême prudence, l'on nous permettra de rapporter la conclusion de ce quinzième chapitre; nous citons :

« Dans de telles circonstances, il n'est pas étonnant que l'avenir du français et de l'anglais constitue un défi à relever pour les uns et pour les autres et que la question linguistique, avec toutes ses ramifications, demeure le sujet de tensions permanentes dans la vie politique, sociale et culturelle du Québec des prochaines décennies » (p. 448).

Vingt-huit chapitres ou vingt-huit articles? L'objectif central était clairement rapporté dans l'avant-propos par Gérard Daigle : «La recherche d'une vision globale » de la société québécoise (p. 17). La façon de procéder était elle aussi fort bien circonscrite, nous l'avons déjà rapporté. Je confirmerais les références expressément fortes aux publications (a) de Jean-Charles Falardeau Essai sur le Québec contemporain (1953), publié aux Presses de l'Université Laval ; b) de Marcel Rioux et Yves Martin La société canadienne française (1969), publié sous le titre French Canadian Society chez Mc Clelland and Stewart Toronto et c) de Gérard Boismenu, Laurent Maihot et Jacques Rouillard Le Québec en textes (1986), publié aux Éditions du Boréal à Montréal.

Or, le Québec n'a ni introduction, ni conclusion. Il n'a pas l'unité qu'avaient les publications de Jean-Charles Falardeau, de Marcel Rioux et d'Yves Martin. Manquent une ligne directive, une problématique centrale susceptible de regrouper et d'articuler des contributions fort diverses et c'est pourquoi nous préférons faire état de vingt-huit articles plutôt que de vingt-huit chapitres relevant d'un seul et même livre. Le Québec en jeu n'est pas non plus assimilable à « l'anthologie » publié par Gérard Boismenu, Laurent Mailhot et Jacques Rouillard dans la mesure où il ne s'agit pas de «morceaux choisis » rédigés au cours des ans et sélectionnés à posteriori, mais d'articles spécifiques rédigés pour les besoins de la cause.

Mission impossible : nous avons déjà fait état de la dispersion de ces disciplines qui se donnent pour objet l'étude de la société, qu'elle soit ou non québécoise. Il se pourrait cependant que le caractère de plus en plus pluraliste, diversifié, voire éclaté et 
conflictuel du Québec d'aujourd'hui puisse ajouter à cette dispersion un défi particulièrement difficile à relever.

Bernard Solasse

\section{Université Laval}

Histoire des idées sociologiques. Tome 1 : Des origines à Weber, par Michel Lallemant, Paris, Nathan, coll. «circa » $\mathrm{N}^{\circ} 22,1993,256 \mathrm{p}$., ISBN 2-09-190-095-8.

Histoire des idées sociologiques. Tome 2 : De Parsons aux contemporains, par Michel Lallemant, Paris, Nathan, coll. "circa " $\mathrm{N}^{\circ} 30,1993,254$ p., ISBN 2-09-190-096-6.

On remarque depuis le début des années 1990 une prolifération significative d'ouvrages introductifs qui permettent à ceux qui veulent aborder la sociologie de trouver une initiation complète à la discipline. Ainsi, trois ouvrages intitulés Histoire de la sociologie paraissent en l'espace d'un an chez trois prestigieux éditeurs parisiens : celle de Pierre-Jean Simon aux Presses universitaires de France (1991); celle de Friedrich Jonas chez Larousse (1991, édition originale allemande en 1965), celle de Charles-Henri Cuin et de François Guesle (parue en deux tomes chez La Découverte en 1992). Par ailleurs, tandis que l'on met à jour des manuels déjà éprouvés, comme Essentials of Sociology, de Brinkerhoff et al. ( $2^{\mathrm{e}}$ édition, St-Paul, West Pub., 1992) aux États-Unis, ou Introduction à la sociologie de Michel De Coster ( $3^{\mathrm{e}}$ édition, Bruxelles, De Boeck, 1992) en Belgique, on édite de nouveaux manuels de base, comme le récent Traité de sociologie, d'orientation actionniste, publié aux Presses universitaires de France (1992), et dirigé par Raymond Boudon. Mais une revue complète de ces nouveautés demanderait en soi une chronique beaucoup plus élaborée, comme l'ont fait par exemple Serge Paugam dans la Revue française de sociologie (Paris, Presses du CNRS, Octobre 1993, XXXIV-4, p. 657-671), et Louis Moreau De Bellaing dans la revue L'Homme et la société, Éd. L'Harmattan, $\mathrm{N}^{\circ} 107$, janvier 1993, p. 187-193).

En fait, comme le soulignait Robert Nisbet au début de son livre La Tradition sociologique (PUF, 1984, p. 15; $1^{\text {re }}$ édition américaine en 1966), il existe deux façons de présenter l'histoire de la pensée : soit en étudiant les grands penseurs (comme l'ont réussi Raymond Aron dans Les Grandes étapes de la pensée sociologique, paru chez Gallimard en 1967; et Pierre Bourdieu et al. dans Le Métier de sociologue, édité l'année suivante chez Mouton), soit en abordant directement les principaux concepts de la discipline, comme dans la plupart des manuels du genre. On pourrait de même ajouter les nombreux recueils de textes choisis, comme celui de Karl von Meter, paru dernièrement chez Larousse (coll. «Textes essentiels », 1992, 851 p.). Cependant, l'étude de l'évolution des idées d'une discipline donnée correspond peut-être à une tendance plus nouvelle, comme le prouve la parution récente d'une Histoire de la pensée sociologique, par Gilles Ferréol (Éd. A. Colin, 1994), à ne pas confondre avec le présent ouvrage.

L'approche de cette Histoire des idées sociologiques de Michel Lallemant ajoute à cette subdivision habituelle. Comme l'ensemble des titres parus jusqu'à maintenant 